\title{
First approach to UAV-based contact inspection: a smart payload for navigation in the neighbourhood of structures
}

\author{
L. M. González-de Santos ${ }^{1 *}$, J. Martínez-Sánchez ${ }^{1}$, H. González-Jorge ${ }^{2}$, A. Novo ${ }^{1}$, P. Arias ${ }^{1}$. \\ ${ }^{1}$ Applied Geotechnologies Group, Dept. Natural Resources and Environmental Engineering, \\ School of Mining and Energy Engineering, University of Vigo, Campus Lagoas-Marcosende, CP 36310 Vigo, Spain \\ (luismgonzalez, joaquin.martinez, annovo, parias)@uvigo.es \\ ${ }^{2}$ Applied Geotechnologies Group, Dept. Natural Resources and Environmental Engineering, School of Aerospace Engineering, \\ University of Vigo, Campus Lagoas, CP 32004 Ourense, Spain. \\ higiniog@uvigo.es \\ Commission IV, WG IV/5
}

KEY WORDS: Unmanned aerial systems, industrial inspection, non-destructive testing, navigation, flight control.

\begin{abstract}
:
Many inspection tasks of structures are already carried out by unmanned aerial vehicles (UAV). Most of these inspections consist of using payloads for close range remote sensing purposes (i.e. digital cameras, thermal or LiDAR sensors). In all these inspection tasks the UAV system does not need to be close to the structure and typically the GPS coverage is good to perform mission navigation. In this paper, a smart payload developed for navigation in the neighbourhood of structures is presented. With this payload the UAV system is able to control the distance to a structure and the angle formed by the UAV and the structure in the horizontal plane. This payload has been calibrated in order to determine the calibration curve and measure the accuracy of the payload. The system has been tested in an indoor environment (GPS-denied). Good position and angular results has been obtained.
\end{abstract}

\section{INTRODUCTION}

During the life cycle of a structure, monitoring its state is essential in order to prevent possible structural failures (Nair, 2006). This makes the inspection and maintenance tasks crucial, being a large part of the investment (Bribián et al. 2009). The costs of these operations increase in structures with difficult accessibility, such as bridges (Ko \& Ni, 2005), dams (Karastathis et al. 2002) or wind farms (Kusiak \& Li, 2011). In the last case, these operations represent a significant investment (Nilsson \& Bertling, 2007), optimizing such tasks is critical for the profitability of the wind farm.

Several non-destructive inspection methods (Rens, Wipf, \& Klaiber, 1997) have been designed for structural inspection tasks. For example, vibration data can be used for location and measurement of damage in structures (Ratcliffe, 2000). Other example is ultrasonic guided waves (Rose, 2002).

All this data can be storaged in a BIM system (Motawa \& Almarshad, 2013), in order to facilitate the use of structural monitoring algorithms (Achenbach, 2009).

\section{RELATED WORK}

Nowadays, the use of UAV systems for inspections tasks is very extended. Non-contact sensor systems, such as cameras or LiDAR sensors, are often used as payload for these tasks, where the UAV do not need to be close to a structure. Padró et al. uses a UAV system with a multispectral camera for supporting opencast mining and restoration monitoring (Padró et al. 2019). Gillins et al. presented a cost-effective bridge inspection using a UAV, where a RGB-camera is used to detect some damaged areas in structural joints of bridges (Gillins, Gillins, \& Parrish,
2016). In these cases, the UAV uses GPS systems for positioning control.

In other cases, the UAV needs to fly in a GPS-denied area, creating the demand of other positioning systems. Several positioning systems have been developed for specific study cases. Araar et al. presents a positioning system composed of a camera with image processing algorithm to detect and follow power lines (Araar \& Aouf, 2014) in order to navigate above them. Something similar is done by Ellemberg et al., where some targets have been placed in the structure and the UAV. It uses image processing algorithms and those targets to navigate around the structure (Ellenberget al. 2014). Omari et al. uses stereoscopic camera and MEMS inertial sensors to estimate the position of the $\mathrm{UAV}$ as well as to create a dense $3 \mathrm{D}$ point cloud for calculating navigable areas in a discretized environment, that is a $3 \mathrm{D}$ space segmented in voxels of application-dependent size (González-de Santos et al. 2018), that may be used for collision avoidance (Omari et al. 2014).

In indoor environments, the most used positioning systems are visual odometry and SLAM (Simultaneous Localization and Mapping), but in outdoor environments this positioning systems do not work well in some cases. Visual Odometry (VO) algorithms generally use a camera pointing towards the ground and obtains the position based on the texture in the image. Hence, one of the main drawbacks in industrial environment is that floors do not use to have enough texture to work with VO. SLAM algorithms use a LiDAR sensor and the geometry of the environment for localization, but in outdoors environments (i.e. navigation near to a dam) there is not enough geometrical information in the sensor range, making this positioning system not suitable. 
In this paper a smart payload for navigation in the neighbourhood of a structure is presented. This payload consists of a series of distance sensors to calculate the height and pose relative to the structure. This payload can be adapted to some different flight controllers and UAV configurations.

\section{METHODOLOGY}

For this paper a quadrotor DJI F450 with a Pixhawk Flight Controller is used. The control software used is PX4, which is an open software flight controller. The height control is done using a LiDAR sensor in the bottom of the UAV geared towards the floor and a IMU (Inertial Measurement Unit). In this case, this sensor is directly connected to the flight controller. The pose control relative to the structure is done using two distance systems separated a fixed distance. Each one of these distance system is composed by different distance sensors with different ranges to get a wider range of distance measurements.

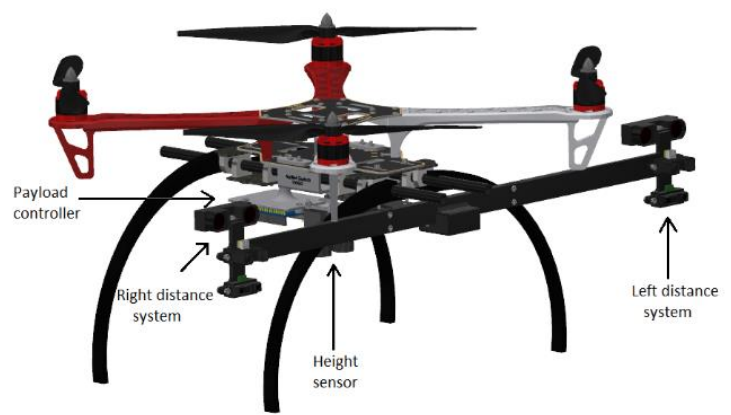

Figure 1. Payload CAD design

With these two distances, the payload is able to calculate the angle and distance relative to the structure. This pose information is used by the controller of the payload to act on the flight controller according to the instructions received. This action is calculated by a PID control algorithm, using as setpoint the data received from the RC transmitter (Figure 2).

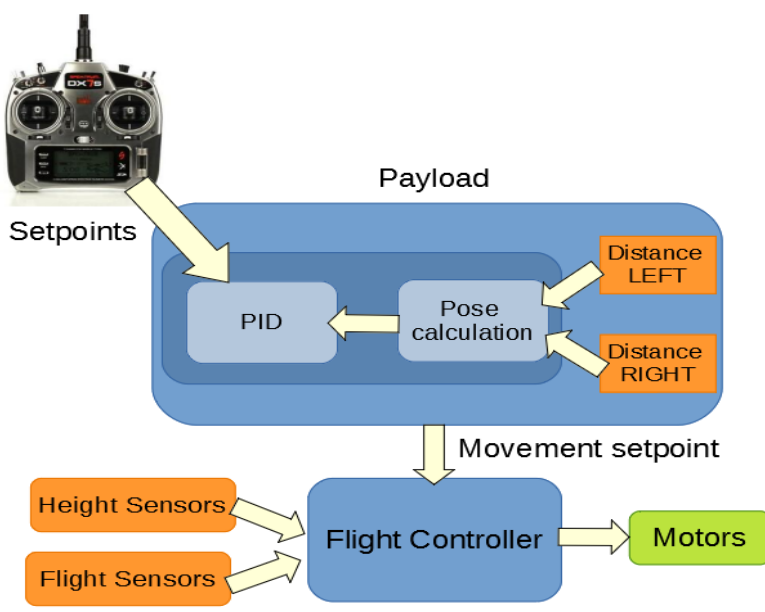

Figure 2. Control diagram

The payload control algorithms are executed in the controller of the payload instead of in the flight controller, because in this way the payload can be adapted to any commercial flight controller. The flight controller handles the stability of the UAV While the payload handle the positioning and the calculation of the necessary movements to achieve the setpoint, making it independent of the frame and configuration of the UAV.

\subsection{Payload design}

Two defined parts can have been distinguished in the payload design. The first one is the mechanical design, where the main objective is to reduce the weight and increase rigidity and vibration absorption. The second one is the electronic design, where the distance sensors have been selected and the PCBs (Printed Circuit Board) have been designed to connect the different electronic components.

\subsubsection{Mechanical design}

As aforementioned, the main objective in the design of the mechanicals parts of the payloads is to reduce the weight and increase rigidity and vibration absorption. In this case, all this designed parts have been made by $3 \mathrm{D}$ printing as proof of concept to test the design. 3D printing has several advantages, since it is easy to use and makes possible to make models in an easy and fast way. But also adds some problems, such as the maximum piece size, which is a problem derived from the print volume of each $3 \mathrm{D}$ printer.

In this case, all the mechanical parts designed are supporting sections that allow us to join the sensors to the UAV frame. As will be seen later, if the distance between the sensors of each side increased, the calculated angle relative to the structure resolution also increased. With this in mind, the support has been designed with the greatest distance between sensors possible. Due to the print volume of the 3D printer used, the distance between sensors in the CAD design is $0.5 \mathrm{~m}$ (Figure 3 ). However, due to the printing tolerances, the real distance is $0.505 \mathrm{~m}$.

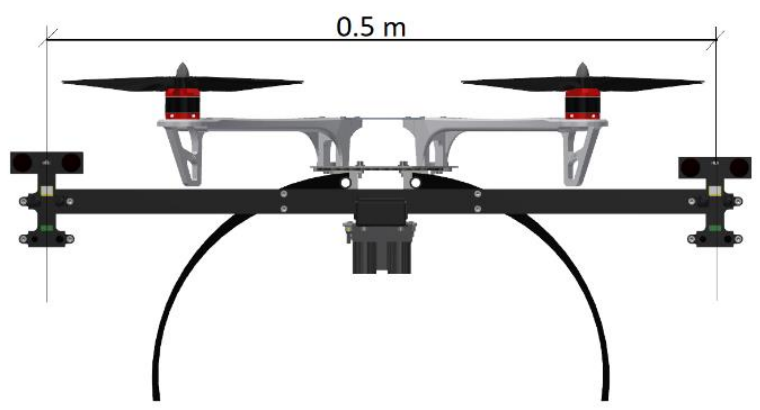

Figure 3. CAD assembly of the Payload in the UAV frame

Due to the 3D printer used, the support has been divided in 4 parts. The different parts of the payload have been made using PLA as material for 3D printing. This material is a very flexible polymer. The pieces made with this material flexible. To make the payload more rigid, a series of fiberglass tubes have been added. In this way, the payload is more rigid and the joints between pieces are stronger (Figure 4). 


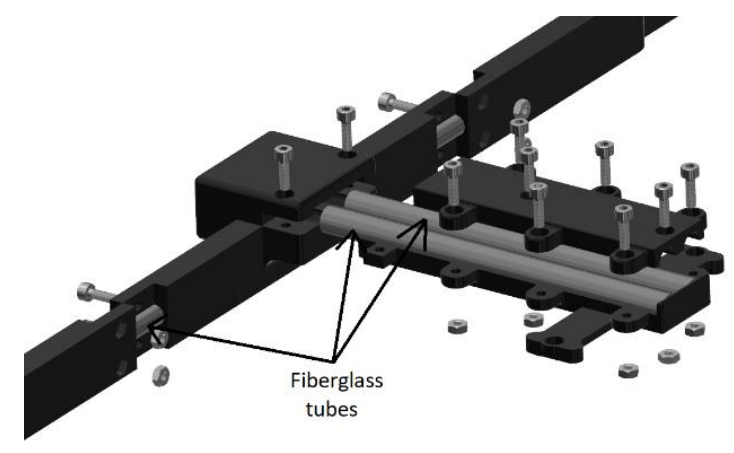

Figure 4. Presentation view of the Payload

\subsubsection{Electronic design}

There are many different sensors on the market, with different technologies for measurement and different ranges and resolution. In this case, Sharp triangulation laser sensors have been selected because they a cheaper than other sensors and have a good resolution. The problem of this kind of sensors is that they have a limited range of measurement and it is smaller than the range of other kind of sensors. To solve this, three different distance sensors with different ranges are used to achieve a greater range, which means that the total range is the sum of the range of each sensor.

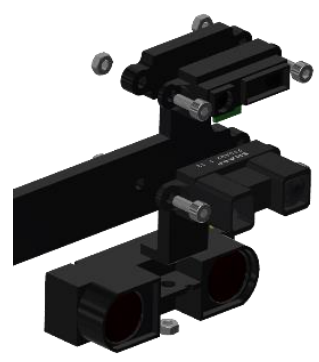

a)

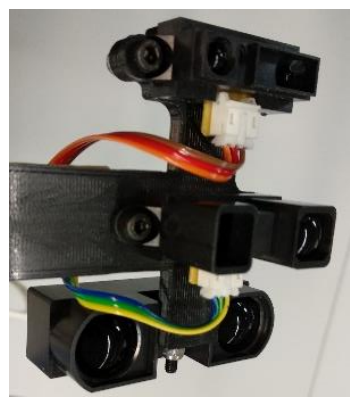

b)
Figure 5. Measurement system composed of three different distance sensors. (a) CAD design. (b) Real system

As can be seen in Table 1, with the three sensors, the distance meter system can measure in a continuous range from $4 \mathrm{~cm}$ to $150 \mathrm{~cm}$. For the distance measurement in the overlap zone between sensors, the midpoint of the overlap is set as a limit for selecting the appropriate measurement. For example, the sensor GP2Y0A41SK0F will have a range in the system from $4 \mathrm{~cm}$ to $25 \mathrm{~cm}$, and the GP2Y0A02YK0F sensor range would start at $25 \mathrm{~cm}$.

These three sensors use an analog output, so to calculate the distance an analog input have to be used in the payload controller. The output of this sensors is not linear, so the calibration curve given in the datasheet of each sensor has been linearized.

\begin{tabular}{lcc}
\hline Sensor & Min. Range $(\mathrm{cm})$ & Max. Range $(\mathrm{cm})$ \\
\hline GP2Y0A41SK0F & 4 & 30 \\
GP2Y0A02YK0F & 20 & 150
\end{tabular}

\begin{tabular}{ccc} 
GP2Y0A710K0F & 100 & 150 \\
\hline Table 1. Model and range of each sensor
\end{tabular}

An Arduino UNO has been used as controller of the payload. Several PCBs have been designed in order to connect the different electronic components of the payload. In this case, the communication between the payload controller and the flight controller is made using a PPM (Pulse Position Modulation) protocol, simulating the signals of the RC transmitter.

\subsection{Payload calibration}

With the distances measured by the sensors from each side of the payload and the distance between them, the angle and the distance relative between the UAV and the structure can be calculated. In Figure 6 shows the relationship between the distance measured by each sensor and the angle and distance of the UAV relative to the structure.

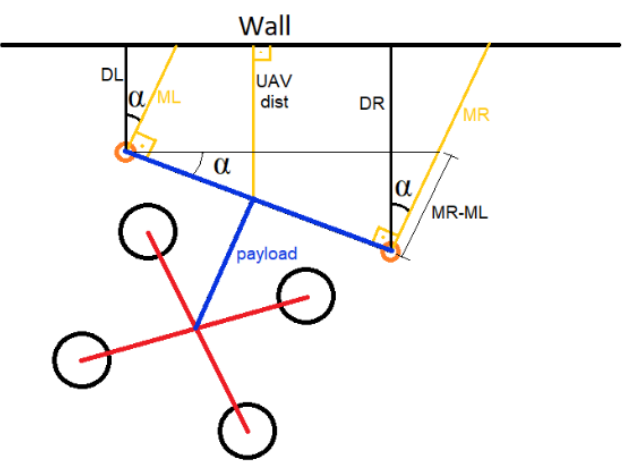

Figure 6. Trigonometric relationship between the measured distances and the UAV pose relative to the wall.

The angle relative to the wall can be calculated using the distance measured by the right sensor $\left(M_{R}\right)$, the distance measured by the left sensor $\left(M_{L}\right)$ and the distance between the sensors (Base) (Equation 1).

$$
\tan (\alpha)=\frac{M_{R}-M_{L}}{\text { Base }}
$$

The origin of coordinates system has been placed in the center of the payload, in the piece that joints the sensors. Therefore, the relative distance between the UAV and the wall is measured perpendicular to the wall and up to this point. To calculate this distance, the distance perpendicular to the wall $\left(D_{L}\right.$ and $\left.D_{R}\right)$ have to be calculated (Equation 2).

$$
\begin{aligned}
& D_{L}=M_{L} * \cos (\alpha) \\
& D_{R}=M_{R} * \cos (\alpha)
\end{aligned}
$$

With this two distance, the distance of the UAV to the wall ( $U A V$ dist) can be calculated using Equation 3:

$$
U A V \text { dist }=D_{L}+\frac{D_{R}-D_{L}}{2}
$$


Once the payload has been assembled and programed, it has been calibrated. For this, a Leica TS15 Robotic Total Station with laser distance measurement has been used. This total station has an angular accuracy of $1 "$ and a distance accuracy of $1 \mathrm{~mm}$. The calibration method consists on compare the angle of the UAV relative to the wall measured by the total station and the angle measured by the payload in order to define the calibration curve of the system (Figure 7).

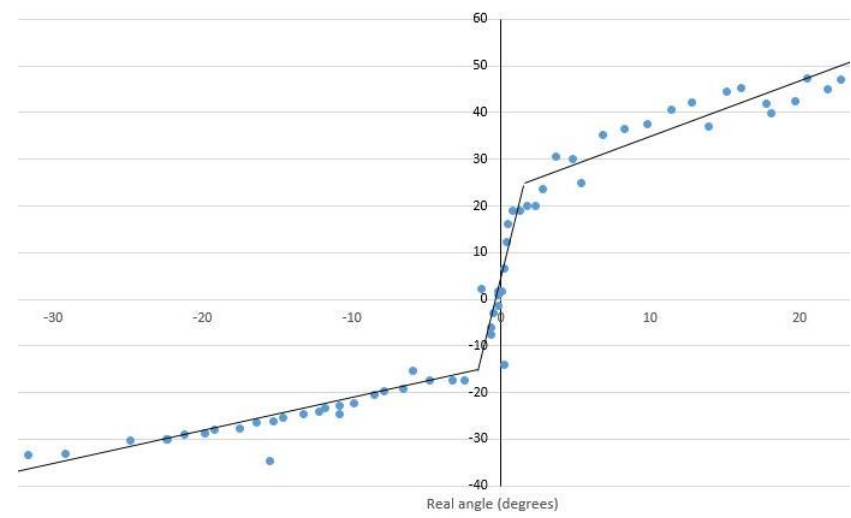

Figure 7. Calibration curve of the sensor

As can be seen in the calibration curve, the system is not able to measure small angles near to $0^{\circ}$. This is due to the distance sensors used, which precision is $1 \mathrm{~cm}$. At angles close to $0^{\circ}$, the measurement of the sensors of each side are almost equal, and given the precision of the sensors, the system is not able to distinguish these angles. This zone of uncertainty, which for the current system is $+/-4^{\circ}$ of the angle measured by the system, is defined by the distance between the sensors.

The calibration curve can be linearized into 3 parts: a central area between $-4^{\circ}$ and $4^{\circ}$ of the angle measured by the system and the two remaining lateral zones.

The distance measurement is based on the sensors reading, which calibration curve is given by the manufacturer in the sensor datasheet, and the angle measured by the system.

\subsection{Position and angular control}

The position and angular control is done using a PID close loop algorithm. Each PID acts on a signal control of the UAV: the PID to control the angular position of the UAV acts on the Yaw signal and the PID to control distance acts on the Pitch signal (Figure 8)

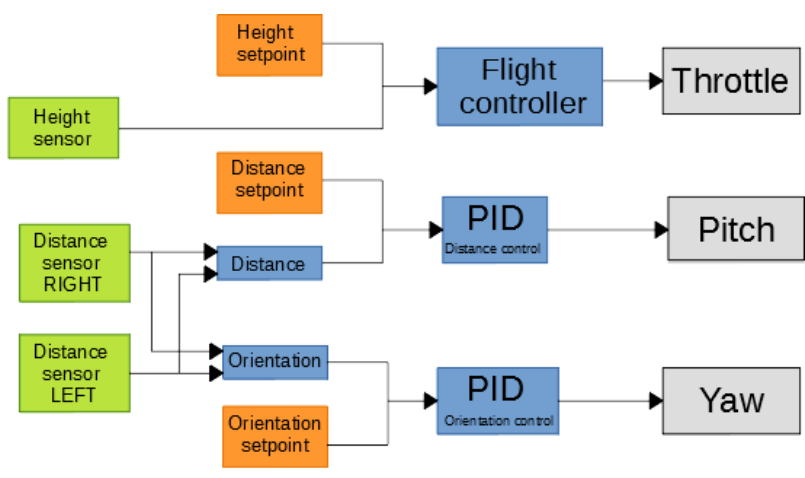

Figure 8. Control scheme

As aforementioned, the system is not suitable to measure angles close to $0^{\circ}$. This makes it infeasible to regulate the angular position for this angles, because the system has not a correct angular state input to the control. To avoid control problems, an angular threshold has been added. When the angular position of the UAV is within the threshold, the PID control does not actuate. The value of this threshold is equal to the zone of uncertainty of the system, that is in this case $+/-4^{\circ}$.

The maximum output of each PID has been limited in order to make the system achieve the setpoint slowly and avoid overshooting.

\section{RESULTS AND DISCUSSION}

\subsection{Experiments and results}

The weight of the payload is 235 grams, which makes it adaptable to small UAV systems. Figure 9 shows the montage of the system on UAV used to test it.

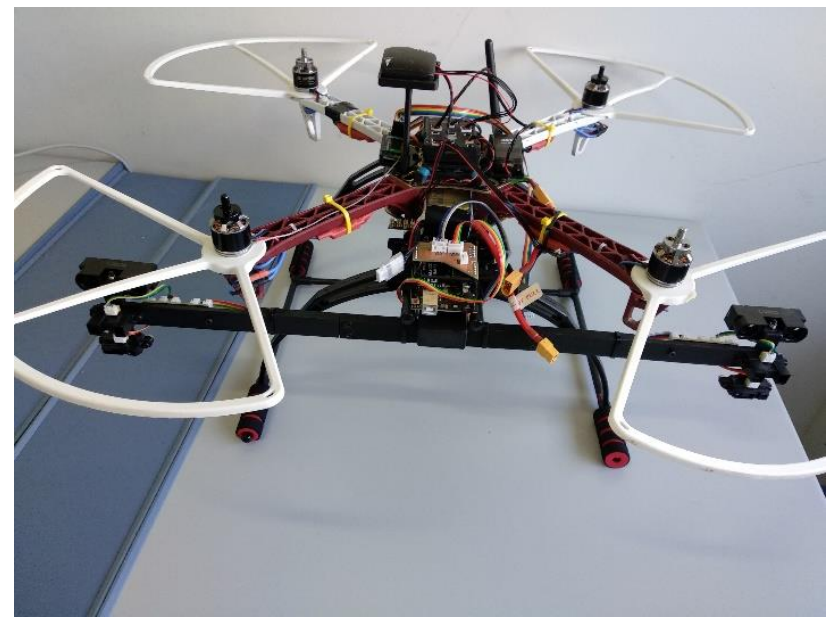

Figure 9. Payload mounted in a DJI F450 frame

The payload has been tested in an indoor laboratory of the Mining and Energy Engineering School at the University of Vigo. Figure 10 shows the response of the angular control system. 


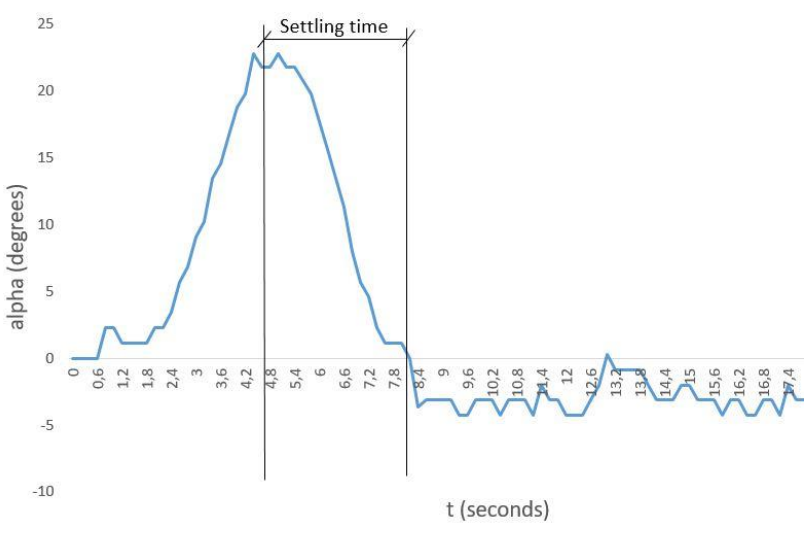

Figure 10. Angular control system response

The system with the current values of control variables has a settling time of 3.5 seconds approximately. The system has an angular positioning error that is due to the threshold that have been added.

\section{CONCLUSIONS}

This paper presents a payload designs for the control of the pose of a UAV relative to a structure in a GPS-denied environment that only use distance sensors. This payload is not feasible to measure angles close to $0^{\circ}$ due to the distance sensors precision and resolution. Despite this, a fitting control has been achieved from the angular data calculated by the system. The angular resolution of the payload can be improved by replacing the actual distance sensors, of negligible cost, with higher resolution distance meters. This would result in a decreasing of the uncertainty range of the angle measurement.

This payload has been developed as the first stage in the development of a system to carry out a contact structure inspection system, where this angular positioning uncertainty is to be absorbed by the mechanical components of the contact system.

\section{ACKNOWLEDGEMENTS}

This project has received funding from the European Union's Horizon 2020 research and innovation programme under grant agreement No 769255. This document reflects only the views of the author(s). Neither the Innovation and Networks Executive Agency (INEA) nor the European Commission is in any way responsible for any use that may be made of the information it contains. Authors would like to thank to the Universidade de Vigo for the financial support $(00 \mathrm{Vl} 131 \mathrm{H} 641.02)$. The statements made herein are solely the responsibility of the authors.

\section{REFERENCES}

Achenbach, J. D. 2009. Structural health monitoring--What is the prescription? Mechanics Research Communications, 36, 137-142.

Araar, O., \& Aouf, N. 2014. Visual servoing of a quadrotor uav for autonomous power lines inspection. 22nd Mediterranean Conference on Control and Automation, (págs. 1418-1424).
Bribián, I. Z., Usón, A. A., \& Scarpellini, S. 2009. Life cycle assessment in buildings: State-of-the-art and simplified LCA methodology as a complement for building certification. Building and Environment, 44, 2510-2520.

Ellenberg, A., Branco, L., Krick, A., Bartoli, I., \& Kontsos, A. 2014. Use of unmanned aerial vehicle for quantitative infrastructure evaluation. Journal of Infrastructure Systems, 21, 04014054.

Ellenberg, A., Kontsos, A., Moon, F., \& Bartoli, I. 2016. Bridge related damage quantification using unmanned aerial vehicle imagery. Structural Control and Health Monitoring, 23, 11681179.

Gillins, M. N., Gillins, D. T., \& Parrish, C. 2016. Cost-effective bridge safety inspections using unmanned aircraft systems (UAS). Geotechnical and Structural Engineering Congress 2016, (págs. 1931-1940).

González-de Santos, L., Díaz-Vilariño, L., Balado, J., MartínezSánchez, J., González-Jorge, H., \& Sánchez-Rodríguez, A. 2018. Autonomous Point Cloud Acquisition of Unknown Indoor Scenes. ISPRS International Journal of GeoInformation, 7, 250.

Karastathis, V. K., Karmis, P. N., Drakatos, G., \& Stavrakakis, G. 2002. Geophysical methods contributing to the testing of concrete dams. Application at the Marathon Dam. Journal of Applied Geophysics, 50, 247-260.

Klein, L., Li, N., \& Becerik-Gerber, B. 2012. Imaged-based verification of as-built documentation of operational buildings. Automation in Construction, 21, 161-171.

Ko, J. M., \& Ni, Y. Q. 2005. Technology developments in structural health monitoring of large-scale bridges. Engineering structures, 27, 1715-1725.

Kusiak, A., \& Li, W. 2011. The prediction and diagnosis of wind turbine faults. Renewable energy, 36, 16-23.

Motawa, I., \& Almarshad, A. 2013. A knowledge-based BIM system for building maintenance. Automation in Construction, 29, 173-182.

Nair, R. S. 2006. Preventing disproportionate collapse. Journal of performance of constructed facilities, 20, 309-314.

Nilsson, J., \& Bertling, L. 2007. Maintenance management of wind power systems using condition monitoring systems - life cycle cost analysis for two case studies. IEEE Transactions on energy conversion, 22, 223-229.

Omari, S., Gohl, P., Burri, M., Achtelik, M., \& Siegwart, R. 2014. Visual industrial inspection using aerial robots. Proceedings of the 2014 3rd International Conference on Applied Robotics for the Power Industry, (págs. 1-5). 
Padró, J.-C., Carabassa, V., Balagué, J., Brotons, L., Alcañiz, J. M., \& Pons, X. 2019. Monitoring opencast mine restorations using Unmanned Aerial System (UAS) imagery. Science of The Total Environment, 657, 1602-1614.

Pagnano, A., Hópf, M., \& Teti, R. 2013. A roadmap for automated power line inspection. Maintenance and repair. Procedia Cirp, 12, 234-239.

Ratcliffe, C. P. 2000. A frequency and curvature based experimental method for locating damage in structures. Journal of vibration and acoustics, 122, 324-329.

Rens, K. L., Wipf, T. J., \& Klaiber, F. W. 1997. Review of nondestructive evaluation techniques of civil infrastructure. Journal of performance of constructed facilities, 11, 152-160.

Rose, J. L. 2002. A baseline and vision of ultrasonic guided wave inspection potential. Journal of pressure vessel technology, 124, 273-282.

Wardhana, K., \& Hadipriono, F. C. 2003. Analysis of recent bridge failures in the United States. Journal of performance of constructed facilities, 17, 144-150. 